\title{
A NOTE ON THE PRECEDING PAPER *
}

BY

D. R. CURTISS

The results of Dr. Walsh's paper are so interesting and capable of so many applications that it may be worth while to indicate a briefer proof of his fundamental theorem which connects with earlier work in this field.

First let us note that Walsh's Theorem II is a form of Laguerre's Theorem given below. In fact, if we write

$$
f(z)=\left(z-\alpha_{1}\right)\left(z-\alpha_{2}\right) \cdots\left(z-\alpha_{k}\right),
$$

Walsh's relation

$$
\frac{1}{z-\alpha_{1}}+\frac{1}{z-\alpha_{2}}+\cdots+\frac{1}{z-\alpha_{k}}=\frac{k}{z-\alpha}
$$

becomes

$$
\frac{f^{\prime}(z)}{f(z)}=\frac{k}{z-\alpha}
$$

or

$$
\alpha=z-k \frac{f(z)}{f^{\prime}(z)} .
$$

Walsh's Theorem II states that if the roots of $f(z)$ are in the circular region $C$, and if $z$ is exterior to $C$, then $\alpha$ lies in $C$. This is precisely the result obtained by Laguerre as given on page 59 of volume I of his collected works, if expressed in non-homogeneous coördinates as on page 57.

We shall use another form of Laguerre's Theorem (loc. cit., p. 57) to prove the underlying proposition on which Walsh bases his proof of his Theorem I, as follows:

Every circle through any point $z$ and its "derived point" $\alpha$ as defined by (1) either passes through all the roots of $f(z)$, or else has at least one root in the region interior to it, and at least one root in the exterior region.

I have recently called attention $\dagger$ to the fact that this is a corollary of a theorem of Bôcher's on jacobians which has served as a starting point in Walsh's earlier papers.

With a slight change in Walsh's notation, whereby we substitute $z$ 's for $\alpha$ 's, the proposition from which Theorem I is deduced may be stated thus:

* Presented to the Society April 14, 1922, under the title On the zeros of successive polars of a binary form.

† S c i e n c e, vol. 55 (1922), p. 193. 
Let $z_{1}, z_{2}, \cdots, z_{k}$ lie within a circular region $C$, and let $\sum_{r}^{k}$ denote the sum of all products of the $z$ 's taken $r$ at a time. Then the equation

$$
a_{0} z^{k}+k a_{1} z^{k-1}+\frac{k(k-1)}{2 !} a_{2} z^{k-2}+\cdots+k a_{k-1} z+a_{k}=0,
$$

where

$$
-a_{k}=a_{0} \sum_{k}^{k}+a_{1} \sum_{k-1}^{k}+a_{2} \sum_{k-2}^{k}+\cdots+a_{k-1} \sum_{1}^{k}
$$

has at least one root in $C$ for each set of values $a_{0}, a_{1}, \cdots, a_{k-1}$.

This theorem is trivial if all the $a$ 's are zero. In any other case we proceed by induction, assuming our proposition true for $k=r-1$ and proving its validity for $k=r$. It is obviously true for $k=1$.

Let us place $k=r$ in (2) and in (1). The resulting left-hand member of (2) we will use as $f(z)$ in (1). We write

$$
\begin{aligned}
f(z) & =a_{0} z^{r}+r a_{1} z^{r-1}+\cdots+a_{r}, \\
z f^{\prime}(z)-r f(z) & =-r\left[a_{1} z^{r-1}+(r-1) a_{2} z^{r-2}+\cdots+a_{r}\right],
\end{aligned}
$$

so that (1) becomes

$$
\alpha=-\frac{a_{1} z^{r-1}+(r-1) a_{2} z^{r-2}+\cdots+a_{r}}{a_{0} z^{r-1}+(r-1) a_{1} z^{r-2}+\cdots+a_{r-1}} .
$$

Now consider the equation obtained by replacing $\alpha$ in (3) by $z_{r}$; cleared of fractions and arranged according to powers of $z$ this is

(4) $\left(a_{0} z_{r}+a_{1}\right) z^{r-1}+(r-1)\left(a_{1} z_{r}+a_{2}\right) z^{r-2}+\cdots+\left(a_{r-1} z_{r}+a_{r}\right)=0$.

But if we compare this with (2), and note that

$$
\begin{aligned}
&-\left(a_{r-1} z_{r}+a_{r}\right)=-a_{r-1} z_{r}+a_{0} \sum_{r}^{r}+a_{1} \sum_{r-1}^{r} \\
& \quad+a_{2} \sum_{r-2}^{r}+\cdots+a_{r-1} \sum_{1}^{r} \\
&=-a_{r-1} z_{r}+a_{0} z_{r} \sum_{r-1}^{r-1}+a_{1}\left(z_{r} \sum_{r-2}^{r-1}+\sum_{r-1}^{r-1}\right) \\
& \quad \quad+a_{2}\left(z_{r} \sum_{r-3}^{r-1}+\sum_{r-2}^{r-1}\right)+\cdots+a_{r-1}\left(z_{r}+\sum_{1}^{r-1}\right) \\
&=\left(a_{0} z_{r}+a_{1}\right) \sum_{r-1}^{r-1}+\left(a_{1} z_{r}+a_{2}\right) \sum_{r-2}^{r-1} \\
& \quad+\cdots+\left(a_{r-2} z_{r}+a_{r-1}\right) \sum_{1}^{r-1},
\end{aligned}
$$

we see that (4) is a case of (2) for $k=r-1$, and hence (4) has a root $Z_{r}$ in $C$.

We now give $z$ the value $Z_{r}$ in (3) and obtain the result that the "derived point" $\alpha$ is $z_{r}$. By Laguerre's Theorem every circle through $Z_{r}$ and $z_{r}$ either has on it a root of $f(z)$ or has a root in its interior and one exterior to it; but $Z_{r}$ and $z_{r}$ are in $C$, so that we can draw through them a circle interior to $C$. Thus $f(z)$ has a root in $C$, and on completing our induction we see that (2) has a root in $C$. 
The theorem we have just proved can not, however, be regarded as a new one. It is equivalent to the theorem of Grace:*

If a form $f(z)$ is apolar to a given form $\phi(z)$, then it has a zero within any circle enclosing all the roots of $\phi(z)$.

If equation (2) and the equation

$$
\left(z-z_{1}\right)\left(z-z_{2}\right) \cdots\left(z-z_{k}\right)=0
$$

are made homogeneous, the condition that (2) and (5) be apolar is precisely the relation just following (2). Since this holds, Grace's theorem requires that a root of (2) lie within any circle enclosing all the roots of (5), which are $z_{1}, z_{2}, \cdots, z_{k}$.

Grace's theorem is a consequence of Laguerre's when the latter is put in homogeneous form. If in (1) we replace $\alpha$ by $\xi / \eta, z$ by $x / y$, and $f(z)$ by $y^{k} f(x, y)$, (1) takes the form

$$
\xi f_{x}^{\prime}+\eta f_{y}^{\prime}=0
$$

which is the equation of the first polar of $(\xi, \eta)$ with respect to $f(x, y)$. One form of Laguerre's theorem (loc. cit., p. 59) may, then, be thus stated:

If the point $\alpha=\xi / \eta$ is without a circle $C$ containing all the roots of $f$, all the roots of the first polar of $\alpha$ with respect to $f$ are within $C$.

Consider a set of points $z_{1}, z_{2}, \cdots, z_{k}$, and let $f_{1}$ be the first polar of $z_{1}$ with respect to $f$, let $f_{2}$ be the first polar of $z_{2}$ with respect to $f_{1}$, etc. If all these $z$ 's are outside $C$, the roots of all the polynomials $f_{1}, f_{2}, \cdots, f_{k-1}$ are within $C$. If (2) and (5) are apolar, $z_{k}$ is the root of $f_{k-1}$, and Grace's theorem, in which $f$ and $\phi$ may be interchanged, follows directly.

The "mixed polars" $f_{1}, f_{2}, \cdots$ are perhaps worth writing down in another form. Thus if we use the notation, for $r<k$,

$$
\psi(z)=\left(z-z_{1}\right)\left(z-z_{2}\right) \cdots\left(z-z_{r}\right)=c_{0} z^{r}+c_{1} z^{r-1}+\cdots+c_{r},
$$

we have

$$
(-1)^{r} f_{r}(x, y)=c_{r} \frac{\partial^{r} f}{\partial x^{r}}-c_{r-1} \frac{\partial^{r} f}{\partial x^{r-1} \partial y}+\cdots+(-1)^{r} c_{0} \frac{\partial^{r} f}{\partial y^{r}} .
$$

In non-homogeneous notation the equation $f_{r}=0$ becomes

* The zeros of a polynomial, Proceedings of the Cambridge Philos o p hi cal S o c i e t y, vol. 11 (1900-02), p. 352. See also Szegö's paper, Bemerkungen zu einem Satz von J. H. Grace über die Wurzeln algebraischer Gleichungen, $\mathrm{M}$ a th $\mathrm{e} \mathrm{m}$ a t i s c $\mathrm{h}$ e Zeitschrift, vol. 13 (1922), p. 28, in which the "Faltungssatz" is, in a sense, the complement of the theorem proved above. 
D. R. CURTiss.

(7)

$$
\psi \frac{d^{r} f}{d z^{r}}-(k-r+1) \frac{d \psi}{d z} \frac{d^{r-1} f}{d z^{r-1}}+\frac{(k-r+1)(k-r+2)}{2} \frac{d^{2} \psi}{d z^{2}} \frac{d^{r-2} f}{d z^{r-2}}
$$

$$
+\cdots+(-1)^{r} \frac{(k-r+1)(k-r+2) \cdots k}{r !} \frac{d^{r} \psi}{d z^{r}} f=0 .
$$

If every root of $f$ is within a circle $C$, and every root of $\psi$ is without $C$, then every root of (7) is within $C$.

NORTHWESTERR UNIVERSITY,

Evanston, InL. 